PROCEEDINGS OF THE

AMERICAN MATHEMATICAL SOCIETY

Volume 128, Number 11, Pages 3291-3300

S 0002-9939(00)05384-3

Article electronically published on April 28, 2000

\title{
A TYPE OF STRASSEN'S THEOREM FOR POSITIVE VECTOR MEASURES WITH VALUES IN DUAL SPACES
}

\author{
JUN KAWABE
}

(Communicated by Dale Alspach)

\begin{abstract}
In this paper, we extend a type of Strassen's theorem for the existence of probability measures with given marginals to positive vector measures with values in the dual of a barreled locally convex space which has certain order conditions. In this process of the extension we also give some useful properties for vector measures with values in dual spaces.
\end{abstract}

\section{INTRODUCTION}

In a celebrated paper, Strassen 21] gave a necessary and sufficient condition for the existence of probability measures with given marginals. His theorem has been extended by many authors in more general settings (cf. Dudley [3], Edwards [5], Hoffmann-Jørgensen [6], Shortt [18], Skala [19] and so on). The first attempt to extend it to vector measures has been made by März and Shortt [13] and Hirshberg and Shortt [7, and they treat vector measures with values in the positive cone of a Banach lattice of a certain type: the so-called KB-spaces.

In this paper, we extend a type of Strassen's theorem (see, e.g., Theorem 5.2 of [5] and Theorem 1 of [19]) for probability measures to positive vector measures with values in the weak dual of a barreled locally convex space which has certain order conditions (see Section 3 for the precise statement of the order conditions and examples). In this process of the extension we first establish a compactness criterion for a set of vector measures with respect to the topology which is a natural analogy of the usual weak topology of real measures, and then we give the extension (Theorem 1) as its application.

In this paper, all the topological spaces and topological vector spaces are Hausdorff, and the scalar fields of topological vector spaces are taken to be the field $\mathbb{R}$ of real numbers.

Received by the editors July 9, 1998 and, in revised form, December 20, 1998.

2000 Mathematics Subject Classification. Primary 28B05, 28A33; Secondary 46A40.

Key words and phrases. Strassen's theorem, positive vector measure, weak convergence of vector measures, barreled locally convex space, Riesz space.

This research was supported by Grant-in-Aid for General Scientific Research No. 11640160, the Ministry of Education, Science, Sports and Culture, Japan.

(C)2000 American Mathematical Society 


\section{NotATION AND PRELIMINARIES}

Let $S$ be a completely regular space and $\mathcal{B}(S)$ the $\sigma$-field of Borel subsets of $S$. Let $X$ be a locally convex space and $X^{*}$ the topological dual of $X$. Denote by $\left\langle x, x^{*}\right\rangle$ the natural duality between $X$ and $X^{*}$. Let $\xi$ be one of the weak topology $\sigma\left(X^{*}, X\right)$, the Mackey topology $\tau\left(X^{*}, X\right)$ and the strong topology $\beta\left(X^{*}, X\right)$. Denote by $X_{\xi}^{*}$ the space $X^{*}$ with the topology $\xi$, and we write $X_{\sigma}^{*}, X_{\tau}^{*}, X_{\beta}^{*}$ when $\xi=\sigma\left(X^{*}, X\right), \tau\left(X^{*}, X\right), \beta\left(X^{*}, X\right)$, respectively.

A finitely additive set function $\mu: \mathcal{B}(S) \rightarrow X_{\xi}^{*}$ is called a vector measure if it is $\sigma$-additive for the topology $\xi$, i.e., for any sequence $\left\{E_{n}\right\}$ of pairwise disjoint subsets of $\mathcal{B}(S)$, we have $\sum_{n=1}^{\infty} \mu\left(E_{n}\right)=\mu\left(\bigcup_{n=1}^{\infty} E_{n}\right)$ in the topology $\xi$ on $X^{*}$. We say that a vector measure $\mu: \mathcal{B}(S) \rightarrow X_{\xi}^{*}$ is Radon if for each $\varepsilon>0, E \in \mathcal{B}(S)$, and $\xi$-continuous seminorm $p$ on $X^{*}$, there exists a compact subset $K$ of $E$ such that $\|\mu\|_{p}(E-K)<\varepsilon$, where $\|\mu\|_{p}$ denotes the $p$-semivariation of $\mu$ (see Diestel and Uhl [2], Lewis [12, and Kluvánek and Knowles [10] for definitions). Denote by $\mathcal{M}_{t}\left(S ; X_{\xi}^{*}\right)$ the set of all Radon vector measures $\mu: \mathcal{B}(S) \rightarrow X_{\xi}^{*}$. We also denote by $\mathcal{M}_{t}(S)$ the set of all real Radon measures on $S$. Then, $\mathcal{M}_{t}(S)$ is a Banach space with the total variation norm $|m| \equiv|m|(S)$. A subset $\mathcal{V}$ of $\mathcal{M}_{t}\left(S ; X_{\xi}^{*}\right)$ is said to be uniformly bounded if $\sup _{\mu \in \mathcal{V}}\|\mu\|_{p}(S)<\infty$ for every $\xi$-continuous seminorm $p$ on $X^{*}$.

If $\mu$ is a vector measure and $x \in X$, then $x \mu$ defined by $(x \mu)(E) \equiv\langle x, \mu(E)\rangle$, $E \in \mathcal{B}(S)$, is a real measure. Then, the following is obvious:

Fact 1. $\mu \in \mathcal{M}_{t}\left(S ; X_{\sigma}^{*}\right)$ if and only if $x \mu \in \mathcal{M}_{t}(S)$ for each $x \in X$.

By a theorem of Orlicz and Pettis (see, e.g., McArthur [14, Corollary 1] and [12. Theorem 1.1]) and a characterization of regularity of vector measures (see [12, Theorem 1.6]), we have

$$
\mathcal{M}_{t}\left(S ; X_{\sigma}^{*}\right)=\mathcal{M}_{t}\left(S ; X_{\tau}^{*}\right),
$$

and if $X$ is semi-reflexive, that is, $\left(X_{\beta}^{*}\right)^{*}=X$, then they coincide with $\mathcal{M}_{t}\left(S ; X_{\beta}^{*}\right)$, since in this case the Mackey topology is equal to the strong topology on $X^{*}$ (see, e.g., [9, 20.1]). Consequently, whenever we assume that $X$ is semi-reflexive, it is not necessary to distinguish the specific topologies $\sigma\left(X^{*}, X\right), \tau\left(X^{*}, X\right)$ and $\beta\left(X^{*}, X\right)$ concerning the $\sigma$-additivity and the Radonness of vector measures with values in $X^{*}$.

The same is true of the uniform boundedness of vector measures: In this case we assume that $X$ is barreled. Since every $\sigma\left(X^{*}, X\right)$-bounded subset of $X^{*}$ is $\beta\left(X^{*}, X\right)$-bounded, the following is readily proved:

Fact 2. The properties being uniformly bounded for $\sigma\left(X^{*}, X\right), \tau\left(X^{*}, X\right)$, and $\beta\left(X^{*}, X\right)$ are equivalent for any subset $\mathcal{V}$ of $\mathcal{M}_{t}\left(S ; X_{\sigma}^{*}\right)$, and this is the case that $x(\mathcal{V}) \equiv\{x \mu: \mu \in \mathcal{V}\}$ is uniformly bounded for each $x \in X$, i.e., $\sup _{\mu \in \mathcal{V}}|x \mu|(S)<\infty$ for each $x \in X$. Further, the principle of uniform boundedness (see Corollary of III.4.2 of [16]) ensures that the set $x(\mathcal{V})$ is uniformly bounded if and only if $\sup _{\mu \in \mathcal{V}}\left|\int_{S} f d(x \mu)\right|<\infty$ for each bounded, continuous real valued function $f$ on $S$.

In this paper, we need an integral of real valued measurable functions with respect to vector measures with values in locally convex spaces. Let $\mu: \mathcal{B}(S) \rightarrow X_{\xi}^{*}$ be a vector measure. A real valued Borel measurable function $f$ on $S$ is said to be $\mu$-integrable if (a) $f$ is $x \mu$-integrable for each $x \in X$, and (b) for each $E \in \mathcal{B}(S)$, 
there exists an element of $X^{*}$, denoted by $\int_{E} f d \mu$, such that

$$
\left\langle x, \int_{E} f d \mu\right\rangle=\int_{E} f d(x \mu)
$$

for each $x \in X$. This integral is a special case of the integral defined by 12, and we refer the reader to [12, 10] for the properties of this integral. We note here the following:

Fact 3. If $X_{\xi}^{*}$ is sequentially complete (this is satisfied, for instance, if $X$ is barreled and $\xi=\sigma\left(X^{*}, X\right), \tau\left(X^{*}, X\right)$ or $\beta\left(X^{*}, X\right)$; see [16. IV.6.1]), then every bounded, Borel measurable, real valued function on $S$ is $\mu$-integrable.

We now introduce the notion of weak convergence of vector measures. Denote by $C(S)$ the Banach space of all bounded, continuous, real valued functions on $S$ with the norm $\|f\| \equiv \sup _{s \in S}|f(s)|$. Assume that $X_{\xi}^{*}$ is sequentially complete. Let $\left\{\mu_{\alpha}\right\}$ be a net in $\mathcal{M}_{t}\left(S ; X_{\xi}^{*}\right)$ and $\mu \in \mathcal{M}_{t}\left(S ; X_{\xi}^{*}\right)$. We say that $\left\{\mu_{\alpha}\right\}$ converges weakly for the topology $\xi$ to $\mu$, and write $\mu_{\alpha} \stackrel{w}{\longrightarrow} \mu$ for $\xi$, if for each $f \in C(S)$, we have

$$
\int_{S} f d \mu_{\alpha} \rightarrow \int_{S} f d \mu \text { for the topology } \xi .
$$

In the following, we equip $\mathcal{M}_{t}\left(S ; X_{\xi}^{*}\right)$ with the topology determined by this weak convergence and call it the weak topology of vector measures for $\xi$. This topology is a natural analogy of that defined by Dekiert [1 for vector measures with values in Banach spaces, and extends the usual weak topology of real measures (cf. Prokhorov [15], LeCam [11], Varadarajan [24], and Topsøe [22]).

\section{MAin Result}

In this section, we state the main result of this paper and will prove it in the following section. We recall that a vector space $X$ with a partial ordering $\leq$ is an ordered vector space if

(1) $x \leq y$ implies $x+z \leq y+z$ for all $x, y, z \in X$;

(2) $x \leq y$ implies $c x \leq c y$ for all $x, y \in X$ and $c>0$.

A Riesz space is defined to be an ordered vector space such that every pair of elements $x, y$ of $X$ has a supremum $x \vee y$ and an infimum $x \wedge y$. An element $x \in X$ is said to be positive if $x \geq 0$. We say that an ordered vector space is of type $(R)$ if for each $x \in X$, we can find two positive elements $x^{+}$and $x^{-}$of $X$ with $x=x^{+}-x^{-}$. Riesz spaces are of type $(R)$. See Example 3 in the end of this section for other ordered vector spaces of type $(R)$. We refer the reader to the books of Schaefer [16] and Kelley and Namioka 9] for further information on ordered vector spaces and Riesz spaces.

An element $x^{*} \in X^{*}$ is said to be positive if $\left\langle x, x^{*}\right\rangle \geq 0$ for any positive element $x \in X$. We say that a vector measure $\mu \in \mathcal{M}_{t}\left(S ; X_{\sigma}^{*}\right)$ is positive if $\mu(E)$ is a positive element in $X^{*}$ for any $E \in \mathcal{B}(S)$. Then it is easy to prove that $\mu \in \mathcal{M}_{t}\left(S ; X_{\sigma}^{*}\right)$ is positive if and only if $\int_{S} f d(x \mu) \geq 0$ for any positive $x \in X$ and any $f \in C(S)$ with $f \geq 0$. Denote by $\mathcal{M}_{t}^{+}\left(S ; X_{\sigma}^{*}\right)$ the set of all positive vector measures in $\mathcal{M}_{t}\left(S ; X_{\sigma}^{*}\right)$ and we write $\mathcal{M}_{t}^{+}(S)$ instead of $\mathcal{M}_{t}^{+}(S ; \mathbb{R})$.

Given two completely regular spaces $S$ and $T, \pi_{S}$ and $\pi_{T}$ denote the projections $S \times T \rightarrow S$ and $S \times T \rightarrow T$, respectively. For a vector measure $\gamma \in \mathcal{M}_{t}\left(S \times T ; X_{\sigma}^{*}\right)$, 
we define its marginals $\pi_{S}(\gamma)$ and $\pi_{T}(\gamma)$ by $\pi_{S}(\gamma)(A)=\gamma\left(\pi_{S}^{-1}(A)\right)$ and $\pi_{T}(\gamma)(B)=$ $\gamma\left(\pi_{T}^{-1}(B)\right)$ for all $A \in \mathcal{B}(S)$ and $B \in \mathcal{B}(T)$.

The following theorem, which is the main result in this paper, extends a type of Strassen's theorem to positive vector measures with values in the weak dual of a barreled locally convex space which is an ordered vector space of type $(R)$.

Theorem 1 (cf. [5], [19]). Let $S$ and $T$ be completely regular spaces and let $X$ be a barreled locally convex space which is an ordered vector space of type $(R)$. Assume that $\Gamma$ is a uniformly bounded, non-empty convex subset of $\mathcal{M}_{t}^{+}\left(S \times T ; X_{\sigma}^{*}\right)$ which is closed for the weak topology of vector measures for $\sigma\left(X^{*}, X\right)$. In order that there exists a $\gamma \in \Gamma$ with given marginals $\mu \in \mathcal{M}_{t}^{+}\left(S ; X_{\sigma}^{*}\right)$ and $\nu \in \mathcal{M}_{t}^{+}\left(T ; X_{\sigma}^{*}\right)$, i.e., $\pi_{S}(\gamma)=\mu$ and $\pi_{T}(\gamma)=\nu$, it is necessary and sufficient that for every $\left\{f_{i}\right\}_{i=1}^{n} \subset$ $C(S),\left\{g_{i}\right\}_{i=1}^{n} \subset C(T)$ and $\left\{x_{i}\right\}_{i=1}^{n} \subset X$, we have

$$
\sum_{i=1}^{n}\left\langle x_{i}, \int_{S} f_{i} d \mu+\int_{T} g_{i} d \nu\right\rangle \leq \sup \left\{\sum_{i=1}^{n}\left\langle x_{i}, \int_{S \times T}\left(f_{i} \oplus g_{i}\right) d \lambda\right\rangle: \lambda \in \Gamma\right\}
$$

Here $\left(f_{i} \oplus g_{i}\right)(s, t) \equiv f_{i}(s)+g_{i}(t)$ for all $(s, t) \in S \times T$.

Remark 2. When $X$ is reflexive, the existing measure $\gamma \in \Gamma$ in Theorem 1 is countably additive and Radon for the strong topology $\beta\left(X^{*}, X\right)$ since in this case $\mathcal{M}_{t}\left(S \times T ; X_{\sigma}^{*}\right)=\mathcal{M}_{t}\left(S \times T ; X_{\beta}^{*}\right)$ as noted in Section 2 .

Example 3. (1) The following (a)-(g) are barreled locally convex spaces which are Riesz spaces, and hence of type $(R)$ :

(a) The Banach lattice $L_{p}(\Omega, \mathcal{A}, m)$ with a measurable space $(\Omega, \mathcal{A}, m)$ and the Banach lattice $l_{p}(1 \leq p \leq \infty)$. Then $L_{p}(\Omega, \mathcal{A}, m)^{*}=L_{q}(\Omega, \mathcal{A}, m)$ and $l_{p}{ }^{*}=$ $l_{q}(1 \leq p<\infty, 1 / p+1 / q=1)$.

(b) The Banach lattice $C(S)$ with a Hausdorff space $S$. See Theorems IV.6.2 and 6.3 of Dunford-Schwartz [4] for the topological dual of $C(S)$.

(c) The Banach lattice $\mathcal{M}(\Omega)$ of all real measures on a measurable space $(\Omega, \mathcal{A})$.

(d) Let $S$ be a $\sigma$-compact and locally compact Hausdorff space. Denote by $\mathcal{C}(S)$ the space of all real continuous functions on $S$. We endow $\mathcal{C}(S)$ with the topology generated by the family of seminorms $p_{K}$ given by $f \mapsto p_{K}(f)=\sup _{s \in K}|f(s)|(K$ varies in the family of all compact subsets of $S$ ). Then $\mathcal{C}(S)$ is a Fréchet space which is a Riesz space.

(e) Let $S$ be a locally compact Hausdorff space. Denote by $\mathcal{C}_{00}(S)$ the space of all real continuous functions on $S$ with compact support. For any fixed compact subset $K$ of $S$, denote by $C_{K}$ the Banach space of functions in $\mathcal{C}_{00}(S)$ that are supported by $K$, with the uniform norm. We endow $\mathcal{C}_{00}(S)$ with the inductive topology generated by the family of Banach spaces $C_{K}$. Then $\mathcal{C}_{00}(S)$ is a barreled locally convex space which is a Riesz space, and the dual $\mathcal{C}_{00}(S)^{*}$ is the space of all real Radon measures on $S$ (see [16, pp.57-58]).

(f) Let $\mathbb{R}^{\infty}$ be the Fréchet-Montel space of all real sequences with the topology of simple convergence. Let $\mathbb{R}_{0}^{\infty}$ be the Montel space of all real sequences which have only a finite number of non-zero coordinates with the topology of uniform convergence on compact sets. We endow those spaces with the canonical coordinatewise order. Then they are Riesz spaces and we have that $\left(\mathbb{R}^{\infty}\right)^{*}=\mathbb{R}_{0}^{\infty}$ and $\left(\mathbb{R}_{0}^{\infty}\right)^{*}=\mathbb{R}^{\infty}$. 
(g) Let $\Lambda(P)$ be the Köthe sequence space with a Köthe set $P$. Then it is a Fréchet space, provided that $P$ is countable, and a Riesz space under the canonical coordinatewise order (see Jarchow [8, pages 27, 50,69 and 497] for definition and properties). Especially, the Fréchet-Montel space $(s)$ of all rapidly decreasing sequences is a Riesz space and the dual $(s)^{*}$ is the space of all slowly increasing sequences.

(2) We present here some examples which are not Riesz spaces but of type $(R)$. Let $H$ be a real Hilbert space with the inner product $(\cdot, \cdot)$. Denote by $\mathcal{L}_{s}(H)$ and $\mathcal{C}_{s}(H)$ the Banach spaces of all bounded self-adjoint operators on $H$ and of all completely continuous self-adjoint operators on $H$ with the usual operator norm. We also denote by $\mathcal{T}_{s}(H)$ and $\mathcal{S}_{s}(H)$ the Banach space of all trace class self-adjoint operators on $H$ with the trace norm and the Hilbert space of all Hilbert-Schmidt class self-adjoint operators on $H$ with the Hilbert-Schmidt norm. We endow those spaces with the order defined by the relation " $A \leq B \Leftrightarrow(A x, x) \leq(B x, x)$ for all $x \in H$ ". For any $A \in \mathcal{L}_{s}(H)$, put $|A|=\left(A^{2}\right)^{1 / 2}, A^{+}=(|A|+A) / 2$ and $A^{-}=(|A|-A) / 2$. Then they are positive operators on $H$. If $A$ belongs to $\mathcal{L}_{s}(H), \mathcal{C}_{s}(H), \mathcal{T}_{s}(H)$ and $\mathcal{S}_{s}(H)$, then so do $|A|, A^{+}$and $A^{-}$, and we have $A=A^{+}-A^{-}$. Consequently, the spaces above are ordered vector spaces of type $(R)$ and we have that $\mathcal{C}_{s}(H)^{*}=\mathcal{T}_{s}(H), \mathcal{T}_{s}(H)^{*}=\mathcal{L}_{s}(H)$ and $\mathcal{S}_{s}(H)^{*}=\mathcal{S}_{s}(H)$. See Schatten [17] for details.

\section{Proof of Theorem 1}

We need several results, which seem to be of interest by themselves, to prove Theorem 1. Let $S$ be a completely regular space, $X$ a locally convex space, and $\mu \in \mathcal{M}_{t}\left(S ; X_{\sigma}^{*}\right)$. Then we can define a continuous linear operator $T_{\mu}: C(S) \rightarrow X_{\sigma}^{*}$ by

$$
T_{\mu}(f)=\int_{S} f d \mu, \quad f \in C(S),
$$

which is called the operator determined by $\mu$. The following proposition, which may be known, insists that every continuous linear operator, satisfying some tightness condition, from $C(S)$ into the weak dual $X_{\sigma}^{*}$ of a barreled locally convex space $X$ can be determined by a vector measure $\mu \in \mathcal{M}_{t}\left(S ; X_{\sigma}^{*}\right)$.

Proposition 4 (cf. [4, 12, [20]). Let $S$ be a completely regular space and $X$ a barreled locally convex space. Assume that a continuous linear operator $T: C(S) \rightarrow$ $X_{\sigma}^{*}$ satisfies the following tightness condition $\left(^{*}\right)$ : For each $\varepsilon>0$ and $x \in X$, there exists a compact subset $K$ of $S$ such that $|\langle x, T(f)\rangle| \leq \varepsilon\|f\|$ for all $f \in C(S)$ with $f(K)=0$.

Then, there exists a unique vector measure $\mu \in \mathcal{M}_{t}\left(S ; X_{\sigma}^{*}\right)$ such that

$$
T(f)=\int_{S} f d \mu \quad \text { for all } f \in C(S) \text {. }
$$

Proof. Let $T: C(S) \rightarrow X_{\sigma}^{*}$ be a continuous linear operator satisfying the condition $\left.{ }^{*}\right)$. We note that the bidual of $X_{\sigma}^{*}$ is equal to $X^{*}$ since $X$ is barreled. Then, by [9] 21.8], the second adjoint $T^{* *}: C(S)^{* *} \rightarrow X^{*}$ exists and it is an extension of $T$.

Fix $x \in X$ for a moment. By $\left({ }^{*}\right)$, for each $\varepsilon>0$, there exists a compact subset $K$ of $S$ such that

$$
\left|\left\langle f, T^{*}(x)\right\rangle\right|=|\langle x, T(f)\rangle| \leq \varepsilon\|f\|
$$


for all $f \in C(S)$ with $f(K)=0$. Since $T^{*}(x) \in C(S)^{*}$, by Theorem 2 of [20] we can find a real Radon measure $m_{x} \in \mathcal{M}_{t}(S)$ such that

$$
\langle x, T(f)\rangle=\left\langle f, T^{*}(x)\right\rangle=\int_{S} f d m_{x}=\theta\left(m_{x}\right)(f)
$$

for all $f \in C(S)$, where $\theta: \mathcal{M}_{t}(S) \rightarrow C(S)^{*}$ is the natural embedding defined by

$$
\theta(m)(f)=\int_{S} f d m, \quad m \in \mathcal{M}_{t}(S), f \in C(S) .
$$

Since $f \in C(S)$ and $x \in X$ are arbitrary, it follows from (4.1) that

$$
T^{*}(x)=\theta\left(m_{x}\right)
$$

for all $x \in X$.

Fix $E \in \mathcal{B}(S)$ and put

$$
\varphi_{E}(\theta(m))=m(E)
$$

for all $m \in \mathcal{M}_{t}(S)$. Since $\theta: \mathcal{M}_{t}(S) \rightarrow C(S)^{*}$ is an isometric isomorphism, it is easy to see that $\varphi_{E}$ is a bounded linear functional on the linear subspace $\theta\left(\mathcal{M}_{t}(S)\right)$ of $C(S)^{*}$. Therefore, by the Hahn-Banach theorem, there exists an extension $\tilde{\varphi}_{E} \in$ $C(S)^{* *}$ of $\varphi_{E}$. Define the set function $\mu: \mathcal{B}(S) \rightarrow X_{\sigma}^{*}$ by

$$
\mu(E) \equiv T^{* *}\left(\tilde{\varphi}_{E}\right)
$$

for all $E \in \mathcal{B}(S)$. Then it is well-defined, i.e., if $\tilde{\tilde{\varphi}}_{E}$ is another extension of $\varphi_{E}$, then we have $T^{* *}\left(\tilde{\varphi}_{E}\right)=T^{* *}\left(\tilde{\tilde{\varphi}}_{E}\right)$. For, by (4.2) we have $T^{*} X \subset \theta\left(\mathcal{M}_{t}(S)\right)$, and hence

$$
\left\langle x, T^{* *}\left(\tilde{\varphi}_{E}\right)\right\rangle=\left\langle T^{*}(x), \tilde{\varphi}_{E}\right\rangle=\left\langle T^{*}(x), \varphi_{E}\right\rangle=\left\langle T^{*}(x), \tilde{\tilde{\varphi}}_{E}\right\rangle=\left\langle x, T^{* *}\left(\tilde{\tilde{\varphi}}_{E}\right)\right\rangle
$$

holds for all $x \in X$, and this implies that $T^{* *}\left(\tilde{\varphi}_{E}\right)=T^{* *}\left(\tilde{\varphi}_{E}\right)$.

In the following, we shall show that $\mu \in \mathcal{M}_{t}\left(S ; X_{\sigma}^{*}\right)$ and $T$ can be determined by $\mu$. By (4.2), (4.3) and (4.4), for each $x \in X$ and $E \in \mathcal{B}(S)$, we have

$$
m_{x}(E)=\tilde{\varphi}_{E}\left(T^{*}(x)\right)=\left\langle T^{*}(x), \tilde{\varphi}_{E}\right\rangle=\left\langle x, T^{* *}\left(\tilde{\varphi}_{E}\right)\right\rangle=\langle x, \mu(E)\rangle=(x \mu)(E),
$$

and this implies that

$$
x \mu=m_{x}
$$

for all $x \in X$. Since $m_{x} \in \mathcal{M}_{t}(S)$, we have $\mu \in \mathcal{M}_{t}\left(S ; X_{\sigma}^{*}\right)$ by Fact 1 ,

Since $X$ is barreled, $X_{\sigma}^{*}$ is quasi-complete, and so it is sequentially complete. Hence, by Fact 3 every bounded, Borel measurable, real valued function on $S$ is integrable with respect to $\mu$. Consequently, by (4.1) and (4.5) we have

$$
\langle x, T(f)\rangle=\left\langle f, \theta\left(m_{x}\right)\right\rangle=\langle f, \theta(x \mu)\rangle=\int_{S} f d(x \mu)=\left\langle x, \int_{S} f d \mu\right\rangle
$$

for all $x \in X$ and $f \in C(S)$, and this implies that $T$ is determined by $\mu$. Finally, the proof of the uniqueness of $\mu$ is easy.

A net $\left\{\mu_{\alpha}\right\}$ in $\mathcal{M}_{t}\left(S ; X_{\xi}^{*}\right)$ is said to be compact for $\xi$ if every subnet of $\left\{\mu_{\alpha}\right\}$ has a subnet converging weakly for $\xi$. We also say that a net $\left\{m_{\alpha}\right\}$ in $\mathcal{M}_{t}(S)$ is compact if every subnet of $\left\{m_{\alpha}\right\}$ has a subnet which converges in the usual weak convergence of real measures. The following gives a general compactness criterion for a set of vector measures with values in the dual space. 
Proposition 5. Let $S$ be a completely regular space and $X$ a barreled locally convex space. Assume that a net $\left\{\mu_{\alpha}\right\}$ in $\mathcal{M}_{t}\left(S ; X_{\sigma}^{*}\right)$ is uniformly bounded, and for each $x \in X$, the net $\left\{x \mu_{\alpha}\right\}$ is compact in $\mathcal{M}_{t}(S)$. Then $\left\{\mu_{\alpha}\right\}$ is compact in $\mathcal{M}_{t}\left(S ; X_{\sigma}^{*}\right)$ for $\sigma\left(X^{*}, X\right)$. When $X$ is a Montel space, it is also compact for $\beta\left(X^{*}, X\right)$.

Proof. For each $\alpha$, we define a continuous linear operator $T_{\alpha}: C(S) \rightarrow X_{\sigma}^{*}$ by

$$
T_{\alpha}(f)=\int_{S} f d \mu_{\alpha}, \quad f \in C(S)
$$

Denote by $\mathcal{L}\left(C(S), X_{\sigma}^{*}\right)$ the space of all continuous linear operators from $C(S)$ into $X_{\sigma}^{*}$, and by $\mathcal{L}_{\sigma}\left(C(S), X_{\sigma}^{*}\right)$ the same space with the topology of simple convergence. We also denote by $\left(X_{\sigma}^{*}\right)^{C(S)}$ the set of all mappings from $C(S)$ into $X_{\sigma}^{*}$. Put $\mathcal{H}=\left\{T_{\alpha}\right\}$ and denote by $\mathcal{H}_{1}$ the closure of $\mathcal{H}$ in $\left(X_{\sigma}^{*}\right)^{C(S)}$ for the topology of simple convergence. By the uniform boundedness of $\left\{\mu_{\alpha}\right\}$, for each $f \in C(S)$, the set $\left\{T_{\alpha}(f)\right\}$ is bounded, and hence relatively compact in $X_{\sigma}^{*}$ since $X$ is barreled (see IV.5.2. of [16]). From this and Tychonoff's theorem it follows that $\mathcal{H}_{1}$ is compact in $\left(X_{\sigma}^{*}\right)^{C(S)}$. To prove that $\mathcal{H}$ is a relatively compact subset of $\mathcal{L}_{\sigma}\left(C(S), X_{\sigma}^{*}\right)$, we have only to show that $\mathcal{H}_{1} \subset \mathcal{L}\left(C(S), X_{\sigma}^{*}\right)$. Since the set $\left\{\left\langle x, T_{\alpha}(f)\right\rangle\right\}$ is bounded for each $x \in X$ and $f \in C(S)$ as stated above, it follows from the Banach-Steinhaus theorem (see, e.g., [16, Theorem III.4.2]) that $\mathcal{H}$ is an equicontinuous subset of $\mathcal{L}\left(C(S), X_{\sigma}^{*}\right)$. Then $\mathcal{H}_{1} \subset \mathcal{L}\left(C(S), X_{\sigma}^{*}\right)$ by III.4.3 of [16]. Thus, we have finished the proof of the relative compactness of $\mathcal{H}$, and hence for any subnet $\left\{\mu_{\alpha^{\prime}}\right\}$ of $\left\{\mu_{\alpha}\right\}$, we can find a subnet $\left\{\mu_{\alpha^{\prime \prime}}\right\}$ of $\left\{\mu_{\alpha^{\prime}}\right\}$ and an operator $T \in \mathcal{L}\left(C(S), X_{\sigma}^{*}\right)$ such that

$$
\langle x, T(f)\rangle=\lim _{\alpha^{\prime \prime}}\left\langle x, T_{\mu_{\alpha^{\prime \prime}}}(f)\right\rangle=\lim _{\alpha^{\prime \prime}}\left\langle x, \int_{S} f d \mu_{\alpha^{\prime \prime}}\right\rangle
$$

for all $x \in X$ and $f \in C(S)$.

We now show that $T$ satisfies tightness condition $\left({ }^{*}\right)$ of Proposition 4 Fix $\varepsilon>0$ and $x \in X$. By (4.6), we have

$$
|\langle x, T(f)\rangle|=\lim _{\alpha^{\prime \prime}}\left|\left\langle x, \int_{S} f d \mu_{\alpha^{\prime \prime}}\right\rangle\right|=\lim _{\alpha^{\prime \prime}}\left|\int_{S} f d\left(x \mu_{\alpha^{\prime \prime}}\right)\right|
$$

for all $f \in C(S)$. On the other hand, since $\left\{x \mu_{\alpha}\right\}$ is compact in $\mathcal{M}_{t}(S)$ by assumption, there exist a subnet $\left\{m_{\alpha^{\prime \prime \prime}}\right\}$ of $\left\{x \mu_{\alpha^{\prime \prime}}\right\}$ and a real measure $m \in \mathcal{M}_{t}(S)$ such that

$$
m_{\alpha^{\prime \prime \prime}} \stackrel{w}{\longrightarrow} m .
$$

Since $m$ is Radon, there exists a compact subset $K$ of $S$ such that

$$
|m|(S-K)<\varepsilon .
$$

Fix $f \in C(S)$ with $f(K)=0$. Then, it follows from (4.7), (4.8) and (4.9) that

$$
\begin{aligned}
|\langle x, T(f)\rangle| & =\lim _{\alpha^{\prime \prime \prime}}\left|\int_{S} f d m_{\alpha^{\prime \prime \prime}}\right|=\left|\int_{S} f d m\right| \\
& =\left|\int_{S-K} f d m\right| \leq\|f\| \cdot|m|(S-K)<\varepsilon\|f\|,
\end{aligned}
$$

and this implies that $T$ satisfies condition $\left(^{*}\right)$ of Proposition 4. Consequently, by Proposition 4 we can find a vector measure $\mu \in \mathcal{M}_{t}\left(S ; X_{\sigma}^{*}\right)$ such that

$$
T(f)=\int_{S} f d \mu
$$


for all $f \in C(S)$. Hence, by (4.6) we have

$$
\lim _{\alpha^{\prime \prime}}\left\langle x, \int_{S} f d \mu_{\alpha^{\prime \prime}}\right\rangle=\left\langle x, \int_{S} f d \mu\right\rangle,
$$

and this implies that $\left\{\mu_{\alpha}\right\}$ is compact in $\mathcal{M}_{t}\left(S ; X_{\sigma}^{*}\right)$ for $\sigma\left(X^{*}, X\right)$.

Assume that $X$ is a Montel space. Then, by Proposition 34.6 of [23] the strong and weak topologies coincide on the bounded subsets of $X_{\beta}^{*}$, and equivalently, on the bounded subsets of $X_{\sigma}^{*}$ since $X$ is barreled. From this and the uniform boundedness of $\left\{\mu_{\alpha}\right\}$ it follows that $\left\{\mu_{\alpha}\right\}$ is also compact for $\beta\left(X^{*}, X\right)$.

Remark 6. By Fact 2 and a well known compactness criterion for real measures (see Theorem 2a of [20]), the assumption of Proposition 5 is satisfied if we assume that for each $x \in X$, the net $\left\{x \mu_{\alpha}\right\}$ is uniformly bounded and uniformly tight.

The following generalizes Lemma 5.1 of Hoffmann-Jørgensen [6].

Proposition 7. Let $S$ and $T$ be completely regular spaces and let $X$ be a barreled locally convex space which is an ordered vector space of type $(R)$. Assume that a net $\left\{\gamma_{\alpha}\right\} \subset \mathcal{M}_{t}^{+}\left(S \times T ; X_{\sigma}^{*}\right)$ is uniformly bounded. If $\pi_{S}\left(\gamma_{\alpha}\right) \stackrel{w}{\longrightarrow} \mu \in \mathcal{M}_{t}^{+}\left(S ; X_{\sigma}^{*}\right)$ and $\pi_{T}\left(\gamma_{\alpha}\right) \stackrel{w}{\longrightarrow} \nu \in \mathcal{M}_{t}^{+}\left(T ; X_{\sigma}^{*}\right)$ for $\sigma\left(X^{*}, X\right)$, then every subnet of $\left\{\gamma_{\alpha}\right\}$ has a subnet converging weakly for $\sigma\left(X^{*}, X\right)$ to a vector measure $\gamma \in \mathcal{M}_{t}^{+}\left(S \times T ; X_{\sigma}^{*}\right)$ such that $\pi_{S}(\gamma)=\mu$ and $\pi_{T}(\gamma)=\nu$.

Proof. We first show that for each $x \in X$, the net $\left\{x \gamma_{\alpha}\right\}$ is compact in $\mathcal{M}_{t}(S)$. Fix $x \in X$. Since $X$ is of type $(R), x$ is represented as the difference of two positive elements $x^{+}$and $x^{-}$with $x=x^{+}-x^{-}$. Since each $\gamma_{\alpha}$ is positive, we have $\left\{x^{+} \gamma_{\alpha}\right\} \subset \mathcal{M}_{t}^{+}(S \times T)$, and by assumption it is easy to verify that $\pi_{S}\left(x^{+} \gamma_{\alpha}\right)=$ $x^{+}\left(\pi_{S}\left(\gamma_{\alpha}\right)\right) \stackrel{w}{\longrightarrow} x^{+} \mu$ and $\pi_{T}\left(x^{+} \gamma_{\alpha}\right)=x^{+}\left(\pi_{T}\left(\gamma_{\alpha}\right)\right) \stackrel{w}{\longrightarrow} x^{+} \nu$. Consequently, by the proof of Lemma 5.1 of [6], the net $\left\{x^{+} \gamma_{\alpha}\right\}$ is compact in $\mathcal{M}_{t}(S)$. The compactness of the net $\left\{x^{-} \gamma_{\alpha}\right\}$ follows in the same way. Therefore, we can easily show that the net $\left\{x \gamma_{\alpha}\right\}$ is also compact in $\mathcal{M}_{t}(S)$.

Since $\left\{\gamma_{\alpha}\right\}$ is uniformly bounded by assumption, it follows from Proposition 5 that $\left\{\gamma_{\alpha}\right\}$ itself is compact in $\mathcal{M}_{t}^{+}\left(S \times T ; X_{\sigma}^{*}\right)$ for $\sigma\left(X^{*}, X\right)$, and hence every subnet of $\left\{\gamma_{\alpha}\right\}$ has a subnet converging weakly for $\sigma\left(X^{*}, X\right)$ to a vector measure $\gamma \in$ $\mathcal{M}_{t}^{+}\left(S \times T ; X_{\sigma}^{*}\right)$.

It remains to prove that $\gamma$ has its marginals $\mu$ and $\nu$, i.e., $\pi_{S}(\gamma)=\mu$ and $\pi_{T}(\gamma)=\nu$. For simplicity, we assume that $\gamma_{\alpha} \stackrel{w}{\longrightarrow} \gamma, \pi_{S}\left(\gamma_{\alpha}\right) \stackrel{w}{\longrightarrow} \mu$ and $\pi_{T}\left(\gamma_{\alpha}\right) \stackrel{w}{\longrightarrow}$ $\nu$ for $\sigma\left(X^{*}, X\right)$. Then, by the continuity of the projections $\pi_{S}$ and $\pi_{T}$, we have $\pi_{S}\left(\gamma_{\alpha}\right) \stackrel{w}{\longrightarrow} \pi_{S}(\gamma)$ and $\pi_{T}\left(\gamma_{\alpha}\right) \stackrel{w}{\longrightarrow} \pi_{T}(\gamma)$ for $\sigma\left(X^{*}, X\right)$. Consequently, by the uniqueness of the limit, we have $\pi_{S}(\gamma)=\mu$ and $\pi_{T}(\gamma)=\nu$, and the proof is complete.

We are now ready to prove Theorem 1. The necessity of (3.1) is clear, and so we only prove sufficiency. Assume (3.1) and denote by $M_{\Gamma}$ the set of all pairs $(\varphi, \psi) \in \mathcal{M}_{t}^{+}\left(S ; X_{\sigma}^{*}\right) \times \mathcal{M}_{t}^{+}\left(T ; X_{\sigma}^{*}\right)$ such that there exists a $\gamma \in \Gamma$ with $(\varphi, \psi)$ as its marginals. Then $M_{\Gamma}$ is non-empty and convex.

Denote by $C(S) \otimes X$ the algebraic tensor product of $C(S)$ and $X$, i.e., the linear space of all finite linear combinations of the basic elements $f \otimes x$, which are assigned to the function $s \in S \rightarrow f(s) x$ by the natural embedding from $C(S) \otimes X$ into the space of all $X$-valued continuous functions on $S$. Note that the weak 
topology of vector measures on $\mathcal{M}_{t}\left(S ; X_{\sigma}^{*}\right)$ for $\sigma\left(X^{*}, X\right)$ is just the weak topology $\sigma\left(\mathcal{M}_{t}\left(S ; X_{\sigma}^{*}\right), C(S) \otimes X\right)$ with respect to the natural duality

$$
\left\langle\mu, \sum_{i=1}^{n} f_{i} \otimes x_{i}\right\rangle \equiv \sum_{i=1}^{n} \int_{S} f_{i} d\left(x_{i} \mu\right)
$$

between $\mathcal{M}_{t}\left(S ; X_{\sigma}^{*}\right)$ and $C(S) \otimes X$. This duality is unambiguously defined, i.e., it does not depend on the expression of elements in $C(S) \otimes X$.

The pairing

$$
\langle(\varphi, \psi),(f, g)\rangle=\langle\varphi, f\rangle+\langle\psi, g\rangle
$$

puts the two spaces $\mathcal{M}_{t}\left(S ; X_{\sigma}^{*}\right) \times \mathcal{M}_{t}\left(T ; X_{\sigma}^{*}\right)$ and $(C(S) \otimes X) \oplus(C(T) \otimes X)$ in duality, and we observe that the weak topology

$$
\sigma_{0}=\sigma\left(\mathcal{M}_{t}\left(S ; X_{\sigma}^{*}\right) \times \mathcal{M}_{t}\left(T ; X_{\sigma}^{*}\right),(C(S) \otimes X) \oplus(C(T) \otimes X)\right)
$$

relative to the pairing above is the product of the topologies

$$
\sigma\left(\mathcal{M}_{t}\left(S ; X_{\sigma}^{*}\right), C(S) \otimes X\right) \quad \text { and } \sigma\left(\mathcal{M}_{t}\left(T ; X_{\sigma}^{*}\right), C(T) \otimes X\right)
$$

(see IV.4.2 of [16]). From the Hahn-Banach theorem and the representation theorem of $\sigma_{0}$-continuous linear functionals, it follows that $(\mu, \nu)$ is in the closure of $M_{\Gamma}$ for the weak topology $\sigma_{0}$ above. Consequently, we can find a net $\left\{\left(\varphi_{\alpha}, \psi_{\alpha}\right)\right\}$ of elements in $M_{\Gamma}$ converging weakly for $\sigma_{0}$ to $(\mu, \nu)$, and hence $\varphi_{\alpha} \stackrel{w}{\longrightarrow} \mu$ and $\psi_{\alpha} \stackrel{w}{\longrightarrow} \nu$ for $\sigma\left(X^{*}, X\right)$. Since for each pair $\left(\varphi_{\alpha}, \psi_{\alpha}\right)$ there exists a $\gamma_{\alpha} \in \Gamma$ with marginals $\varphi_{\alpha}$ and $\psi_{\alpha}$, we have $\pi_{S}\left(\gamma_{\alpha}\right)=\varphi_{\alpha} \stackrel{w}{\longrightarrow} \mu$ and $\pi_{T}\left(\gamma_{\alpha}\right)=\psi_{\alpha} \stackrel{w}{\longrightarrow} \nu$ for $\sigma\left(X^{*}, X\right)$. Therefore, by Proposition 7 the net $\left\{\gamma_{\alpha}\right\}$ has a subnet converging weakly for $\sigma\left(X^{*}, X\right)$ to a $\gamma \in \mathcal{M}_{t}^{+}\left(S \times T ; X_{\sigma}^{*}\right)$ such that $\pi_{S}(\gamma)=\mu$ and $\pi_{T}(\gamma)=\nu$. Since $\Gamma$ is closed, we have $\gamma \in \Gamma$, and the proof is complete.

\section{REFERENCES}

[1] M. Dekiert, Kompaktheit, Fortsetzbarkeit und Konvergenz von Vectormaßen, Dissertation, University of Essen, 1991.

[2] J. Diestel and J. J. Uhl, Vector Measures, Amer. Math. Soc. Surveys Vol. 15, Amer. Math. Soc., Providence RI, 1977. MR 56:12216

[3] R. M. Dudley, Real Analysis and Probability, Wadsworth and Brooks/Cole, Pacific Grove, 1989. MR 91g:60001

[4] N. Dunford and J. T. Schwartz, Linear Operators, Part 1: General Theory, John Wiley \& Sons, New York, 1988. MR 90g:47001a]

[5] D. A. Edwards, On the existence of probability measures with given marginals, Ann. Inst. Fourier (Grenoble) 28 (1978), 53-78. MR 81i:28009

[6] J. Hoffmann-Jørgensen, Probability in Banach Spaces, Ecole d'Été de Probabilités de SaintFlour VI-1976, Lecture Notes in Math. 598 (1977), 1-186. MR 57:1595

[7] A. Hirshberg and R. M. Shortt, A version of Strassen's theorem for vector-valued measures, Proc. Amer. Math. Soc. 126 (1998), 1669-1671. MR 98i:28014

[8] H. Jarchow, Locally Convex Spaces, B. G. Teubner, Stuttgart, 1981. MR 83h:46008

[9] J. L. Kelley and I. Namioka, Linear Topological Spaces, Van Nostrand, New York, 1963. MR 29:3851

[10] I. Kluvánek and G. Knowles, Vector Measures and Control Systems, North-Holland, 1976. MR 58:17033

[11] L. LeCam, Convergence in distribution of stochastic processes, Univ. California Publ. Statist. 2 (1957), 207-236. MR 19:128a

[12] D. R. Lewis, Integration with respect to vector measures, Pacific J. Math. 33 (1970), 157-165. MR 41:3706

[13] M. März and R. M. Shortt, Weak convergence of vector measures, Publ. Math. Debrecen 45 (1994), 71-92. MR 96g:28015 
[14] C. W. McArthur, On a theorem of Orlicz and Pettis, Pacific J. Math. 22 (1967), 297-302. MR 35:4702

[15] Yu. V. Prokhorov, Convergence of random processes and limit theorems in probability theory, Theory Probab. Appl. 1 (1956), 177-238. MR 18:943b

[16] H. H. Schaefer, Topological Vector Spaces, Springer-Verlag, New York, 1971. MR 49:7722

[17] R. Schatten, Norm Ideals of Completely Continuous Operators, Springer-Verlag, New York, 1970. MR 41:2449

[18] R. M. Shortt, Strassen's marginal problem in two or more dimensions, Z. Wahrsch. Verw. Gebiete. 64 (1983), 313-325. MR 84i:60023

[19] H. J. Skala, The existence of probability measures with given marginals, Ann. Probab. 21 (1993), 136-142. MR 94f:60004

[20] O. G. Smolyanov and S. V. Fomin, Measures on topological linear spaces, Russian Math. Surveys 31 (1976), 3-56. MR 54:8776

[21] V. Strassen, The existence of probability measures with given marginals, Ann. Math. Statist. 36 (1965), 423-439. MR 31:1693

[22] F. Topsøe, Topology and Measure, Lecture Notes in Math. 133, Springer-Verlag, New York, 1970. MR 54:10546

[23] F. Treves, Topological Vector Spaces, Distributions and Kernels, Academic Press, New York, 1967. MR 37:726

[24] V. S. Varadarajan, Measures on topological spaces, Amer. Math. Soc. Transl. Ser. II 48 (1965), 161-228. MR 26:6342

Department of Mathematics, Faculty of Engineering, Shinshu University, 4-17-1 WAKASATO, NAGANO 380-8553, JAPAN

E-mail address: jkawabe@gipwc.shinshu-u.ac.jp 